\title{
Review
}

\section{Progress on the Prevention and Nursing of Urinary Tract Infection Associated with Radical Hysterectomy and Pelvic Lymphadenectomy}

\author{
Wenyan Yang \\ Shandong Academy of Medical Sciences, Jinan China
}

\section{Keywords}

radical hysterectomy, pelvic lymphadenectomy, urinary tract infection, prevention, progress of nursing

\section{Correspondence}

Wenyan Yang,

E-mail: yangwyjn@126.com

DOI: $10.1515 / i i-2017-0130$

\begin{abstract}
Radical hysterectomy $(\mathrm{RH})$ and pelvic lymphadenectomy are the main treatment methods for early cervical cancer and endometrial carcinoma. Effective care measures, however, can decrease the incidence of UTIs and complications associated with RH and pelvic lymphadenectomy, as well as improve the therapeutic effects of administered drugs and patient prognosis. The writer refers to relevant literatures to analyze the reasons for postoperative UTIs and to provide a brief summary of the nursing methods for and progress in UTI prevention.
\end{abstract}

Radical hysterectomy ( $\mathrm{RH}$ ) and pelvic lymphadenectomy are the main treatment methods for early cervical cancer and endometrial carcinoma. Urinary tract infections (UTIs) are one of the most common early complications of $\mathrm{RH}$ and pelvic lymphadenectomy, with an incidence of approximately $30 \%$ in China and $11 \%-63 \%$ in foreign countries ${ }^{[1,2]}$. UTIs might negatively affect the recovery of bladder functions, extend the length of hospital stay, increase the cost of treatment, and physically and mentally burden patients ${ }^{[3]}$. Effective care measures, however, can decrease the incidence of UTIs and complications associated with $\mathrm{RH}$ and pelvic lymphadenectomy, as well as improve the therapeutic effects of administered drugs and patient prognosis.

\section{Reasons for urinary tract infection}

The analysis of the relevant literature and related factors of postoperative urinary tract infection (UTI) reveals that UTIs occur for the following reasons: First, cervical cancer, endometrial cancer, and other long-term chronic diseases decrease the immunities of patients ${ }^{[4]}$. Second, radical hysterectomy $(\mathrm{RH})$ and pelvic lymphadenectomy may cause considerable pain, thus constricting the surface of the bladder. Consequently, normal blood circulation and contractility decrease, in turn damaging the bladder muscle, some nerves, and sustentacular tissues. These factors are the main reasons for urinary retention, which is more likely to cause UTIs. Collectively, these factors result in a vicious cycle when indwelling preoperative catheter is installed; the installed catheter may cause UTIs. Third, during extended postoperative bed rest, substances accumulate in the lowest point of the posterior wall of the bladder, thus causing UTIs. Fourth, the postoperative catheter is usually indwelled for 10-14 days. Extended indwelling time might increase the probability of UTIs. Under normal circumstances, the urethra is a relatively sterile environment. However, the installation of an indwelling catheter is an invasive operation. If disinfection and surgery are not strictly aseptic, pathogens will ascend to the bladder and cause UTIs. In addition, catheter insertion injures urethral mucosa to some extent, thus damaging the natural barrier of urethral mucosa. Furthermore, the indwelling of a foreign catheter may irritate the urethral and bladder mucosa to different degrees ${ }^{[5]}$, damage the normal physiological environment, and weaken the defense of the urethral mucosa against pathogens. These effects are all risk factors for infection. Fifth, given that the female urethra is short and straight, the urethral sphincter is weak, thus allowing bacteria to easily enter the bladder from the urethra. In addition, the female urethra is close to the vagina and anus and is thus vulnerable to contamination by the normal intestinal parasitic Escherichia 
coli. Contamination by postoperative vaginal secretions is another cause of UTIs. Sixth, the injudicious application of antibiotics increases the likelihood of UTIs. The above factors and care-related factors, such as the poor drainage of the catheter ${ }^{[6]}$, unfulfillment of perineal care, high hanging of the urine bag, delayed dumping of urine, and the frequent replacement of the urine bag and catheter can increase the incidence of UTIs. Related studies have reported on the correlation between the replacement frequency of the urine bag with the results of urine culture ${ }^{[7]}$. The positive rate of urine culture of 10 patients whose urine bags were replaced every day was $61.9 \%$ within one week and $72.4 \%$ within 10 days; whereas those of 40 patients whose urine bags were replaced every week was $0 \%$ and $31.25 \%$ within 10 days. These results indicated that the frequent replacement of urine bags is positively correlated with UTIs. The frequent replacement of the urine bag may destroy the impermeability of urine drainage and cause the contamination of the urine bag and the junction of the catheter end. Furthermore, repeated intubation and urine reflux can increase the incidence of UTIs. Along with the increase of fertility and age, the relaxation of the pelvic floor muscle may cause urinary incontinence, urinary retention, and bladder prolapse. These factors increase the incidence of UTIs.

\section{Progress in the prevention and nursing of UTIs}

\section{Ways to prevent extra-cavity infection}

Extra-cavity infection is influenced by the following aspects: the retention and protection of the catheter; the postoperative cleaning of the perineum and urethral orifice; and the decreased frequency of replacement to prevent damage to the urethral mucosa, urinary tract, and urine bag. Preventive protection measures should be taken around the above points. First, the appropriate catheter should be selected. Silicone gel catheters have good histocompatibility ${ }^{[8]}$ and cause limited irritation to the urinary tract mucosa; moreover, biofilms do not easily form on silicone catheters, thus decreasing the incidence of catheter-related infections [9]. Second, standardize the protective operation of catheter installment. Prior to catheterization, if possible, the patients should clean their own perinea and disinfect their hands in accordance with operating procedures. The catheter should be inserted gently with the appropriate use of lubricants to minimize injury to the urethral mucosa ${ }^{[10]}$. Niu Jialan, et al.
${ }^{[11]}$ found that using $0.5 \%$ iodophor instead of paraffin oil to lubricate the catheter can decrease the incidence of UTIs by $16 \%$ because $0.5 \%$ iodophor has strong antibacterial, sporicidal, antiviral, and antifungal effects. In addition, iodophor does not irritate and or cause allergic reactions in human tissues and mucous membranes. Thus, using iodophor as a lubricant effectively prevents the entry of bacteria into the bladder through the mucous membranes that surround the urinary tract and urethral lumen. When inserting the catheter, use the left hand to hold the labia minora and to keep the catheter away from the perineum and the mucous membranes of the skin outside the meatus urinarius. To keep the catheter sterile, release the left hand to fix the catheter until the catheter is inserted to the required length, thus keeping the meatus urinarius relatively sterile. Third, clean and disinfect the catheter, perineum, and meatus urinarius every day. Scrub the meatus urinarius and perineum with $0.5 \%$ iodophor solution twice a day and increase the frequency of disinfection when secretions increase. Follow the specifications of gynecological perineal scrub. Completely disinfect the meatus urinarius to avoid secondary contamination and disinfect the catheter near the meatus urinarius and the junction of the catheter and drainage bag. In addition, keep the catheter clean to prevent retrograde bacterial infection. Fourth, replace the urine bag and catheter as the patients require. Given that the replacement of the urine bag and catheter is closely related to the incidence of UTIs, the frequency of replacement is essential. Urine should be dynamically monitored and the frequency of replacement should be determined based on the urine $\mathrm{pH}$ value. In general, patients with urine $\mathrm{pH}>6.8$ are at high risk for urethral obstruction and their catheters should be replaced once every two weeks. Patients with urine $\mathrm{pH}<6.17$ are at low risk for urethral obstruction and their catheters should be replaced once every 4 weeks ${ }^{[12]}$. The urine bag should be replaced twice a week. Use $0.5 \%$ iodophor to disinfect the catheter junction to prevent retrograde infection. Fifth, always wash hands.

\section{Control intraluminal infection}

First, a closed drainage system should be used to avoid the separation of the catheter and urine bag during operation ${ }^{[13]}$. Second, instruct patients to drink more water and ensure that they drink more than $2500 \mathrm{ml}$ water daily to achieve physiological rinse and increase urine output ${ }^{[14]}$. Third, decrease bladder rinse; strengthen bladder function via 
exercises to shorten the time of catheter indwelling. Fourth, keep urine drainage clear. During the indwelling period of the catheter, the color, characteristics, and drainage of urine should be closely observed. The bending and pressure of the drainage tube should be avoided to prevent urine bag overflow and ensure smooth drainage. Do not place the urine bag and drainage tube higher than the bladder and keep the urine bag away from the ground to prevent pathogen entry. Fifth, use antimicrobial drugs reasonably. Numerous studies have confirmed that antimicrobial drugs cannot decrease the incidence of UTIs and instead increase the drug resistance of UTI-causing strains. Therefore, observing and knowing the effect of antimicrobial drugs can provide reasonable indications for physicians.

\section{Shorten the time of indwelling catheter}

Numerous studies have shown that the indwelling duration of the catheter is positively correlated with the incidence of UTIs ${ }^{[15]}$. Therefore, personalized care intervention is necessary after $\mathrm{RH}$ and pelvic lymphadenectomy. Training for independent urinary function should be emphasized. At one-week post-surgery, the appropriate time and method of urination should be selected based on the degree of bladder filling and the micturition desire of the patient to restore independent urination as soon as possible. The best time to remove the urinary catheter should be identified to decrease urinary retention and decrease the possibility of re-inserting the urinary catheter and of UTIs. Measure residual urine in the bladder after pulling out the catheter. If the volume of the residual urine exceeds $100 \mathrm{ml}$, use acupuncture treatment once a day. Acupuncture can significantly improve bladder function.

To increase patient compliance, inform the patients and their families of the purpose, fundamentals, and required precautions for indwelling catheters. Correctly guide patients with movement, diet, and hygiene during the indwelling period of the catheter. Provide postoperative intestinal nutrition and parenteral nutrition to enhance the patient's immunity. Guide the patient on bed activity and early bedside activity during the indwelling period of the catheter to decrease the likelihood of UTIs.

In conclusion, the incidence of UTIs associated with $\mathrm{RH}$ and pelvic lymphadenectomy has increased as a result of several interrelated factors: the characteristics of the female genital anatomy; the bacterial colonization of the vagina and perineum; the decreased immunity of patients caused by long-term postoperative bed rest; the large amount of intraoperative blood loss; and damage to the bladder caused by surgery. The analysis of related causes and appropriate nursing measures for UTIs has revealed various risk factors for the incidence of UTIs. This paper provides the basis for the control and avoidance of UTIs in patients who had undergone $\mathrm{RH}$ and lymphadenectomy.

\section{Declarations}

\section{Acknowledgements}

No.

\section{Competing interests}

The author declares that she has no competing interest.

\section{Authors' contributions}

WY Yang made the literature analysis and wrote, discussed and revised the manuscript of this review.

\section{References}

1 Gai L, Le A, Wang Z, et al. Nursing Experience on the Treatment of Laparoscopic Radical Hysterectomy and Pelvic Lymphadenectomy on Uterine Malignancies. Journal of Laparoscopic Surgery, 2010,15 (5): 356,362 .

2 Yan M. Effect of Early Movement on the Recovery of Bladder Function after Radical Hysterectomy. Journal of Youjiang Medical College for Nationalities, 2013, 35 (2): 256-257.

3 Xiao Z, Feng L, Kang S, et al. Evaluation on the Effect of Gynecological Malignancies Postoperative Individualized Nursing Intervention on the Prevention of Urinary Retention. Contemporary Nurses (specialist version), 2011, (2): 64-66.

4 Wang $M$, Chen S, Wang W, et al. Preventive Nursing on Urinary Retention after Radical Hysterectomy of Cervical Cancer. Journal of Guangdong Medical College, 2009,27 (4): 477-478.

5 Li W. Analysis on Nursing of Radical Hysterectomy and Pelvic Lymphadenectomy. Health Vision, 2013,21 (3): 181-182.

6 Qin H. Nursing inside Surgery Room of Radical Hysterectomy. Jilin Medical, 2011,32 (32): 6949-6950.

7 Huang X. Nursing Intervention on Perineal Swelling after Cervical Cancer Radical Surgery. Chinese Journal of Medicine, 2012,14 (7): 1246,1248

8 Liu Y. Clinical Nursing on 28 Cases of Patients with Postoperative Urinary Retention of Cervical Cancer. Qilu Nursing Journal, 2011,17 (8): 99-100.

9 Liao S. Operative Cooperation on Laparoscopic Radical Hysterectomy 
of Cervical Cancer. Health must read, 2012,11 (6): 382-383.

10 Huang A, Yu J, Li Y, et al. Prevention and Nursing of Urinary Retention of Radical Hysterectomy of Cervical Cancer. Fujian Medical Journal, 2011, 33 (5): 157-158.

11 Niu J, Liu Z, Liu X, et al. Clinical Observation on Using Iodophor to Lubrate Catheter to Reduce Urinary Tract Infection. Clinical Nursing Journal, 2006,5 (5): 59-60.

12 Zhao W, Guo X. Effect of Bladder Washout on Cervical Cancer Patients with Postoperative Urinary Retention. Nursing Research, 2011,25 (26):
2412-2412.

13 Li W. Analysis on Postoperative Nursing of Radical Hysterectomy and Pelvic Lymphadenectomy. Uygur Medicine (first half), 2013, (2): 105.

14 Fu Z. Nursing Experience on the Prevention of Urinary Tract Infection Caused by Radical Hysterectomy of Cervical Cancer. Seeking Medicine (Academic Edition), 2012,10 (10): 350.

15 Dai X, Tian M, Li H, et al. Postoperative Prevention and Nursing of Urinary Tract Infection of Cervical Cancer. Chinese Community Physician, 2009,11 (4): 111. 